\title{
Politique
}

Politique

James Iain Gow, Histoire de l'Administration Publique québécoise, 1867-1970, Montréal, Presses de l'Université de Montréal, Toronto, Institut d'administration publique du Canada, 1986, $441 \mathrm{p}$.

\section{Marcel Proulx}

Numéro 13, printemps 1988

Crise de décision

URI : https://id.erudit.org/iderudit/040587ar

DOI : https://doi.org/10.7202/040587ar

Aller au sommaire du numéro

\section{Éditeur(s)}

Société québécoise de science politique

ISSN

0711-608X (imprimé)

1918-6584 (numérique)

Découvrir la revue

Citer ce compte rendu

Proulx, M. (1988). Compte rendu de [James Iain Gow, Histoire de

l'Administration Publique québécoise, 1867-1970, Montréal, Presses de

l'Université de Montréal, Toronto, Institut d'administration publique du

Canada, 1986, 441 p.] Politique, (13), 139-142. https://doi.org/10.7202/040587ar d'utilisation que vous pouvez consulter en ligne.

https://apropos.erudit.org/fr/usagers/politique-dutilisation/ 
James Iain Gow, Histoire de L'Administration Publique québecoise, 1867-1970, Montréal, Presses de l'Université de Montréal, Toronto, Institut d'administration publique du Canada, 1986, $441 \mathrm{p}$.

Cette «Histoire de l'Administration québecoise, 1867-1970» est le fruit de près de vingt ans de recherches, patiemment menées par James Iain Gow et l'équipe d'assistants et d'étudiants à qui il a su faire partager ses intérêts. Ce gigantesque effort de recherche a permis de dépouiller une masse impressionnante d'études, de documents officiels, de rapports internes de l'Administration et de documents d'archives de toutes sortes, fournissant ainsi aux chercheurs qui voudront fouiller davantage certaines des questions abordées dans l'ouvrage une base documentaire solide.

L'auteur a adopté le parti-pris de nous livrer un maximum d'informations sur l'évolution des activités gouvernementales et de l'appareil administratif qui les produit. Il en est résulté un gros ouvrage, assez dense, dont le contenu est d'abord et avant tout descriptif.

Cette description de l'histoire administrative du Québec comporte deux volets parallèles: le premier traite de l'évolution du rôle de l'État et le second du développement du système administratif.

En ce qui regarde le premier volet, la perspective adoptée consiste «à aborder le rôle de l'État par ses productions plutôt que par les déclarations d'intention des gouvernements ou encore les lois adoptées». Cela amène l'auteur à décrire de façon très fine les activités gouvernementales, correspondant à ce que l'on appelle aujourd'hui, dans le jargon administratif, des «programmes».

L'histoire de ces programmes gouvernementaux est divisée en trois grandes périodes: la période 1867-1896, dite de formation 
de l'État québecois; la période 1897-1936, dominée par les fonctions de suppléance et de protection; et enfin la période de 1936 à 1970, marquée par le développement de l'État-providence. Pour chacune de ces périodes, l'auteur décrit systématiquement le contenu des interventions gouvernementales dans les quatre «missions» de l'État, allant dans certains cas jusqu'à la présentation de microactivités.

Après avoir présenté le catalogue des activités gouvernementales à une période donnée, Gow amorce la description du «système administratif» en faisant état du contexte qui a présidé à l'émergence des politiques gouvernementales et administratives du moment. Cette section, qui expose les objectifs et les stratégies des gouvernements en place de même que les contraintes auxquelles ils sont soumis, joue un rôle de charnière dans l'analyse de chacune des périodes de l'histoire administrative du Québec. En effet, elle donne un sens à la fois au volet précédent portant sur les programmes gouvernementaux, et à l'exposé qui suit sur l'organisation et le fonctionnement de l'appareil administratif proprement dit.

Cet appareil administratif, on nous en présente d'abord les structures, puis les grands systèmes de gestion (finances, personnel, services horizontaux, etc.). Le cadre ainsi décrit a un caractère essentiellement formel. Mais l'ouvrage fait malgré tout d'intéressantes incursions dans le domaine des mœurs et des pratiques administratives, à travers surtout la chronique des «scandales administratifs» qui ont marqué chaque époque. On aimerait certes en savoir davantage sur ces aspects informels de la dynamique administrative; mais il ne faut pas en demander trop, sachant que la sociologie de l'administration québecoise contemporaine reste encore très largement inexplorée.

La présentation du système administratif se termine par un exposé fouillé sur la situation des fonctionnaires (effectifs, conditions de carrière, conditions de travail, situation politique). 
Le propos d'ensemble de l'ouvrage est essentiellement de nature inductive, l'auteur tirant, à la fin de chaque chapitre, les conclusions qui se dégagent des phénomènes observés. Celles-ci multiples et éclatées, ne se résument pas en quelques lignes. On notera cependant que plusieurs de ces conclusions mettent en cause des idées reçues concernant le rôle de l'État québécois à certaines époques. Ainsi apprend-on avec intérêt que l'État québécois du $\mathrm{XIX}^{\mathrm{e}}$ siècle intervient de façon relativement marquée dans l'économie à des fins de développement, et ne se confine pas dans le rôle d'État-gendarme. On constate d'ailleurs que l'importance relative des interventions gouvernementales destinées à orienter le développement économique fluctue très largement dans le temps en fonction de la conjoncture politique et économique.

On retiendra aussi la thèse surprenante concernant la continuité des interventions gouvernementales au cours de la période 19361970, considérées comme s'inscrivant dans la même logique de développement de l'État-providence. Si cette façon de faire a le mérite de souligner que, bien avant la Révolution tranquille, on a vu apparaître une série d'interventions gouvernementales caractérisées par le souci de la protection sociale, il me semble que l'on doit malgré tout reconnaître que, qualitativement autant que quantitativement, les interventions gouvernementales au cours de la décennie 1960-1970 se démarquent de celles des deux décennies précédentes.

Soulignons que les conclusions de l'ouvrage débordent le cadre de la vérification des trois hypothèses, élémentaires et à mon avis assez peu intéressantes, énoncées en introduction:

1) l'hypothèse selon laquelle le développement du rôle de l'État au Québec a suivi le modèle général des États occidentaux;

2) l'hypothèse d'une bureaucratisation progressive de l'appareil administratif;

3) et enfin l'hypothèse selon laquelle le rôle de l'État et le 
développement administratif suivent les courbes de l'industrialisation et de l'urbanisation de la société.

En fait, ces hypothèses et les considérations théoriques sur lesquelles elles se fondent paraissent plaquées, dans la mesure où elles s'inscrivent dans une démarche hypothético-déductive que n'emprunte pas vraiment l'auteur, et dont on ne trouve la trace que dans l'introduction et la conclusion. Cela n'enlève rien à la valeur de l'ouvrage, mais cette coquetterie intellectuelle ne lui apporte pas grand-chose non plus.

Cela dit, ce livre est certainement appelé à devenir un ouvrage de référence obligé, aussi bien pour ceux qui s'intéressent à l'histoire politico-administrative du Québec que pour les administrativistes et les spécialistes des politiques gouvernementales.

Marcel Proulx

École nationale d'administration publique

Québec 